\title{
Wiener Index of Graphs with Radius Two
}

\author{
Yin Chen, Baoyindureng Wu, and Xinhui An \\ College of Mathematics and System Science, Xinjiang University, Urumqi, Xinjiang 830046, China
}

Correspondence should be addressed to Baoyindureng Wu; baoyinwu@gmail.com

Received 20 December 2012; Accepted 21 January 2013

Academic Editors: J. Siemons and B. Taeri

Copyright (C) 2013 Yin Chen et al. This is an open access article distributed under the Creative Commons Attribution License, which permits unrestricted use, distribution, and reproduction in any medium, provided the original work is properly cited.

\begin{abstract}
The Wiener index of a graph is the sum of the distances between all pairs of vertices. It has been one of main descriptors that correlate a chemical compound's molecular graph with experimentally gathered data regarding the compound's characteristics. We characterize graphs with the maximum Wiener index among all graphs of order $n$. with radius two. In addition, we pose a conjecture concerning the minimum Wiener index of graphs with given radius. If this conjecture is true, it will be able to answer an open question by You and Liu (2011).
\end{abstract}

\section{Introduction}

Let $G=(V, E)$ be a connected graph. For two vertices $u, v \in$ $V$, the distance $d_{G}(u, v)$ between $u$ and $v$ in $G$ is the length of the shortest path connecting $u$ and $v$ in $G$. The eccentricity $\operatorname{ecc}(v)$ of a vertex $v$ in $G$ is the largest distance from $v$ to another vertex of $G$; that is, $\max \{d(v, w) \mid w \in V(G)\}$. The diameter of $G$ is the maximum eccentricity in $G$, denoted by $\operatorname{diam}(G)$. Similarly, the radius of $G$ is the minimum eccentricity in $G$, denoted by $\operatorname{rad}(G)$. The Wiener index or total distance of $G$, denoted by $W=W(G)$, is the sum of all distances between unordered pairs of distinct vertices of $G$. In other words,

$$
\begin{aligned}
\operatorname{rad}(G) & =\min _{v \in V}\{\operatorname{ecc}(v)\}, \\
\operatorname{diam}(G) & =\max _{v \in V}\{\operatorname{ecc}(v)\}, \\
W(G) & =\sum_{u, v \in V} d(u, v) .
\end{aligned}
$$

The average distance $\mu(G)$ of $G$ is defined as $\mu(G)=$ $W(G) /\left(\begin{array}{c}n \\ 2\end{array}\right)$. A vertex $v \in V(G)$ is called a center of $G$ if $\operatorname{ecc}(v)=\operatorname{rad}(G)$. It is well known that every tree has either exactly one center or two adjacent centers. For the results on Wiener index of trees, we refer to Dobrynin et al. [1], and for some more results on Wiener index of graphs, one can see [2-12].
Plesník [13] addresses a problem on distance of graphs, which remains unresolved.

Problem 1. What is the maximum total or average distance among all graphs of order $n$ with diameter $d$ ?

To see how hard to solve Problem 1, one just consider the following conjecture, due to DeLaViña and Waller [14].

Conjecture 1 (see [14]). Let $G$ be a graph with diameter $d>2$ and order $2 d+1$. Then $W(G) \leq W\left(C_{2 d+1}\right)$, where $C_{2 d+1}$ denotes the cycle of length $2 d+1$.

For any connected $\operatorname{graph} G, \operatorname{rad}(G) \leq \operatorname{diam}(G) \leq 2 \operatorname{rad}(G)$. By considering the close relationship between the diameter and the radius of a graph, it is natural to consider the following problem.

Problem 2. What is the maximum total or average distance among all graphs of order $n$ with radius $r$ ?

By Lemma 3 in Section 2, Problem 2 is more tractable than Problem 1; the graph with maximum total distance among all graphs of order $n$ with radius $r$ must be a tree. But, Problem 2 still seems to be quite challenging. The main focus of this paper is to tackle Problem 2 when the case $r=$ 2 . We will show that the graph with the maximum Wiener index among all connected graphs with radius two is a tree with somewhat fractal-like structure when the number of 
vertices of graphs goes to infinity. It provides some clues for the further investigation of Problem 2 for the case $r \geq 3$. [10].

The following problem remains open, see You and Liu

Problem 3. What is the minimum total or average distance among all graphs of order $n$ with radius $r$ ?

It is easy to see that the complete graph $K_{n}$ has the minimum Wiener index among all connected graphs of order $n$ (and so among all graphs of order $n$ with radius one). For the case of radius two, it is not hard to prove that the extremal graph $G_{n}$ of Problem 3 should be the following: $G_{n} \cong K_{n} \backslash M$, if $n$ is even, where $M$ is a perfect matching; $G_{n} \cong K_{n} \backslash(F \cup\{e\})$, if $n$ is odd, where $F$ is a maximum matching of $K_{n}$ and $e$ is an edge incident with the vertex of degree $n-1$ in $K_{n} \backslash F$.

We denote by $G_{n, r, s}$ the graph obtained in the following way, where $n$ and $r$ are three positive integers with $n \geq 2 r$, $r \geq 3$, and $n-2 r+1 \geq s \geq 1$ : let $u, v, x$, and $y$ be four consecutive vertices on $C_{2 r}$; replace $v$ with a clique $K_{s}$ of order $s$, replace $x$ with a clique of order $n-2 r+2-s$, join each vertex of a clique to all vertices of the other clique, join $u$ to the all vertices of the $K_{s}$, and join $y$ to all vertices of $K_{n-2 r+2-s}$. It is clear that $G_{n, r, s}$ is a graph of order $n$ with radius $r$, and $W\left(G_{n, r, s}\right)=W\left(G_{n, r, s^{\prime}}\right)$ for any $s, s^{\prime} \in\{1, \ldots, r-1\}$.

Conjecture 2. Let $n$ and $r$ be two positive integers with $n \geq$ $2 r$ and $r \geq 3$. For any graph $G$ of order $n$ with radius $r$, $W(G) \geq W\left(G_{n, r, 1}\right)$, with equality if and only if $G \cong G_{n, r, s}$ for $s \in\{1, \ldots, r-1\}$.

\section{Definitions and Lemmas}

Lemma 3. For any connected graph $G$, there exists a spanning tree $T$ of $G$, such that $\operatorname{rad}(T)=\operatorname{rad}(G)$ and $W(T) \geq W(G)$.

Proof. Let $u$ be a center of $G$ and $T$ be a BFS-tree of $G$ rooted at $u$. The tree $T$ is one, as we desired.

The path number $p(G)$ of a graph $G$ is defined to be the maximum cardinality of a subset of vertices that induces a path. Erdös et al. [15] showed that for any connected graph $G$, $p(G) \geq 2 \operatorname{rad}(G)-1$.

Lemma 4. For a tree $G$ with radius $r, 2 \operatorname{rad}(G) \leq p(G) \leq$ $2 \operatorname{rad}(G)+1$.

Proof. Let $P_{u v}$ be the longest path of $G$. Since $G$ is a tree, $p(G)=|V(P)|$. It is clear that a center $w$ of $G$ must be sitting on $P_{u v}, \min \left\{d_{G}(w u), d_{G}(w v)\right\} \geq r-1$ and $\max \left\{d_{G}(w u)\right.$, $\left.d_{G}(w v)\right\} \geq r$. The result then follows.

Lemma 5. Let $T$ be a tree of order $n$ with the maximum Wiener index among all trees with radius two. If $n=4$, then $T \cong P_{4}$, and $p(T)=5$, otherwise.

Proof. If $n=4$, the result is trivial. So, let $n \geq 5$. By Lemma 4 , $4 \leq p(T) \leq 5$. By contradiction, suppose that $p(T)=4$, and let $P=v_{1} v_{2} v_{3} v_{4}$ be a path on four vertices in $T$. Every other vertex not on $P$ must be adjacent to $v_{2}$ or $v_{3}$. Without loss of generality, let $d_{T}\left(v_{2}\right) \leq d_{T}\left(v_{3}\right)$, and let $x$ be a pendent vertex adjacent to $v_{3}$. Set $T^{\prime}=T-v_{2} v_{3}+v_{2} x$. It is easy to check that $W\left(T^{\prime}\right)>W(T)$, contradicting the choice of $T$.

Lemma 6. Let $T$ be a tree. Then

(1) the center of $T$ is a vertex or two adjacent vertices,

(2) the center of $T$ is one vertex if and only if $\operatorname{diam}(T)=$ $2 \operatorname{rad}(T)$.

The above two lemmas assert that if $T$ is a tree of order $n$ with the maximum Wiener index among all trees of order at least five with radius two, then $T$ has the unique center. We denote by $T_{n, t}\left(n_{1}, \ldots, n_{t}\right)$ the tree of order $n$ with radius two and unique center $v_{c}$ such that $d\left(v_{c}\right)=t, N\left(v_{c}\right)=\left\{v_{1}, \ldots\right.$, $\left.v_{t}\right\}$, and $d\left(v_{i}\right)=n_{i}+1$ for each $i$. In particular, $S(n, t)=$ $T_{n, t}\left(n_{1}, \ldots, n_{t}\right)$, with $\left|n_{i}-n_{j}\right| \leq 1$ for any $i, j$.

Lemma 7. Let $T=T_{n, t}\left(n_{1}, \ldots, n_{t}\right)$ be the tree as described above. Then

$$
W(T)=\left(2 n^{2}-5 n+3\right)-(n-2) t-\sum_{i=1}^{t} n_{i}^{2} .
$$

Proof. Let $x_{i}=|\{(u, v) \mid u, v \in V(T), d(u, v)=i\}|$. Hence $W(T)=\sum_{i=1}^{4} i x_{i}$, where

$$
x_{1}=n-1 \text {, }
$$

$$
\begin{gathered}
x_{2}=(n-t-1)+\left(\begin{array}{l}
t \\
2
\end{array}\right)+\sum_{i=1}^{t}\left(\begin{array}{c}
n_{i} \\
2
\end{array}\right), \\
x_{3}=\sum_{i=1}^{t}\left(n-t-1-n_{i}\right)=(t-1) \sum_{i=1}^{t} n_{i}, \\
x_{4}=\sum_{i=1}^{t} \frac{n_{i}\left(n-t-1-n_{i}\right)}{2} .
\end{gathered}
$$

By simple calculation, $W(T)=\left(2 n^{2}-5 n+3\right)-(n-2) t-$ $\sum_{i=1}^{t} n_{i}^{2}$.

The trees $S(n, t)$ and $S(n, t+1)$ when $n=1+t+t^{2}$ are illustrated in Figure 1. We will see that $W(S(n, t))=W(S(n, t+$ 1)) if $n=1+t+t^{2}$ in the next corollary. Note that if $n=$ $1+t+t^{2}$, each vertex in $S(n, t)$, not a pendent vertex, has $t$ children. In this sense, $S(n, t)$ has a fractal-like structure. Our main goal is to prove that if $T$ is a tree of order $n$ with the maximum Wiener index among all trees with radius two, then $T \cong S\left(n, t_{0}\right)$, where $t_{0}$ can be determined. It will be shown in Theorem 9. For smaller value of $n$, one can see that those extremal graphs with radius two in Figure 2, and there exists two extremal graphs when $n=1+t+t^{2}$ for some $t \geq 2$; for instance, see the graphs for $t=2(n=7)$ and $t=3(n=13)$ in Figure 2. 


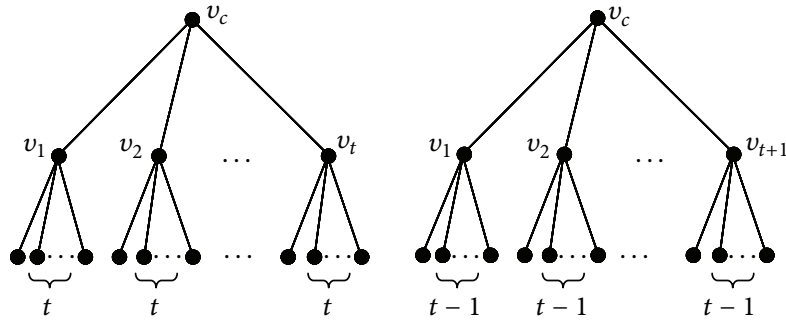

FIgURe 1: The trees $S(n, t)$ and $S(n, t+1)$ when $n=1+t+t^{2}$.

Corollary 8. Let $n$ and $t$ be two positive integers such that $n \geq$ $t+2$ and $1+t+k t \leq n<1+t+(k+1) t$ for some integer $k \geq 0$, and let $p=n-1-t-k t$. Then

$$
\begin{aligned}
W(S(n, t))= & \left(2 t^{2}-t\right) k^{2}+\left[3 t^{2}+(4 p-1) t-2 p\right] k \\
& +\left(t^{2}+3 t p+2 p^{2}-2 p\right),
\end{aligned}
$$

and if $k=t$ and $p=0$, then $W(S(n, t))=W(S(n, t+1))=$ $2 t^{4}+2 t^{3}$.

Proof. By the notation of the previous lemma, In $S(n, t)$, the number of $n_{i}^{\prime} s$ equal to $k+1$ is $p$ and the number of $n_{i}^{\prime} s$ equal to $k$ is $t-p$. So, by Lemma 7 ,

$$
\begin{aligned}
W(S(n, t))= & \left(2 n^{2}-5 n+3\right)-(n-2) t-\sum_{i=1}^{t} n_{i}^{2} \\
= & (2 n-3)(n-1)-(n-2) t \\
& -\left(p(k+1)^{2}+(t-p) k^{2}\right) \\
= & (2 t+2 t k+2 p-1)(t+t k+p) \\
& -(t+t k+p-1) t-\left(2 p k+p+t k^{2}\right) \\
= & \left(2 t^{2}-t\right) k^{2}+\left(3 t^{2}+(4 p-1) t-2 p\right) k \\
& +\left(t^{2}+3 t p+2 p^{2}-2 p\right) .
\end{aligned}
$$

If $k=t$ and $p=0$, by the above formula,

$$
W(S(n, t))=\left(2 t^{2}-t\right) t^{2}+\left(3 t^{2}-t\right) t+t^{2}=2 t^{4}+2 t^{3} .
$$

Moreover, since $1+t+t^{2}=1+(t+1)+(t+1)(t-1)$ and

$$
\begin{aligned}
W(S(n, t+1))= & {\left[2(t+1)^{2}-(t+1)\right](t-1)^{2} } \\
& +\left[3(t+1)^{2}-(t+1)\right](t-1)+(t+1)^{2} \\
= & 2 t^{4}+2 t^{3}
\end{aligned}
$$

this proves $W(S(n, t))=W(S(n, t+1))=2 t^{4}+2 t^{3}$.
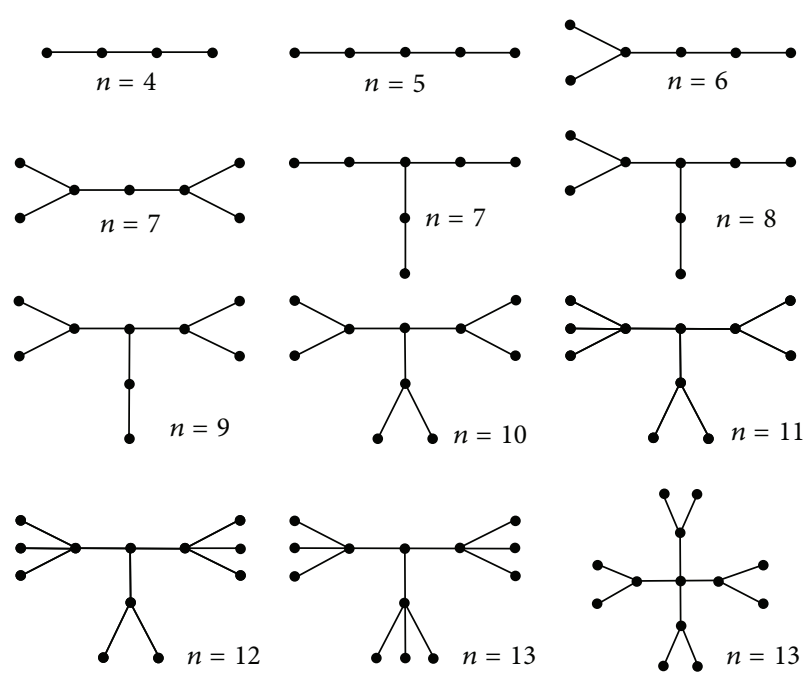

FIGURE 2: The maximum wiener index with given radius two.

\section{Main Results}

For a clear understanding of our main theorem as follows, we make a remark. If $n=1+t_{0}+t_{0}^{2}$ for some positive integer $t_{0}$, then $t_{0}=(\sqrt{4 n-3}-1) / 2$. By Corollary $8, W\left(S\left(n, t_{0}\right)\right)=$ $W\left(S\left(n, t_{0}+1\right)\right)$.

Theorem 9. Let $T$ be a tree with the maximum Wiener index among all trees of order $n$ with radius two. If $n=1+t_{0}+$ $t_{0}^{2}$ for some positive integer $t_{0}$, then $T \in\{S(n,(\sqrt{4 n-3}-$ $1) / 2), S(n,(\sqrt{4 n-3}+1) / 2)\}$, and $T \cong S\left(n, t_{0}\right)$ otherwise, where $t_{0}=\min \left\{i \mid n-1-i-i^{2}<0\right\}$.

Proof. The result is trivial for $n \leq 4$, and assume that $n \geq 5$. By Lemma 5 , let $v_{c}$ be the unique center of $T$, and let $d\left(v_{c}\right)=t$. Set $N\left(v_{c}\right)=\left\{v_{1}, \ldots, v_{t}\right\}$ and $\left|N\left(v_{i}\right) \backslash\left\{v_{c}\right\}\right|=n_{i}$ for $1 \leq i \leq t$. Lemma 7 implies that $\left|n_{i}-n_{j}\right| \leq 1$ for any $i, j$, and thus $T \cong$ $S(n, t)$ for some $t$. To show $t=t_{0}$, as specified in the theorem, it suffices to show that $W(S(n, t))<W\left(S\left(n, t_{0}\right)\right)$ for any $t \neq t_{0}$.

By Corollary 8, $W(S(n, t))=\left(2 n^{2}-5 n+3\right)-(n-2) t-$ $\left[q(k+1)^{2}+(t-q) k^{2}\right]$, where $q=n-1-t-t\lfloor(n-t-1) / t\rfloor$, $0 \leq q \leq t-1$, we have

$$
\begin{aligned}
W(S(n, t)) \leq & \left(2 n^{2}-5 n+3\right)-(n-2) t \\
& -\frac{(n-t-1)^{2}}{t} .
\end{aligned}
$$

Define a function $f(t)=\left(2 n^{2}-5 n+3\right)-(n-2) t-(n-$ $t-1)^{2} / t$, which is an upper bound of $W(S(n, t))$. Its derivative $f^{\prime}(t)=(n-1)^{2} / t^{2}-(n-1)$ has the unique root $t=\sqrt{n-1}$. Observe that

$$
\frac{\sqrt{4 n-3}-1}{2}<\sqrt{n-1} \leq \frac{\sqrt{4 n-3}+1}{2},
$$

and since $t_{0}=\min \left\{i \mid n-1-i-i^{2}<0\right\}$,

$$
\frac{\sqrt{4 n-3}-1}{2}<t_{0} \leq \frac{\sqrt{4 n-3}+1}{2} \text {. }
$$


By the definition of $t_{0}, n=1+t_{0}+t_{0} k+p$, where $t_{0}-2 \leq$ $k \leq t_{0}$ and $0 \leq p \leq t_{0}-1$, and moreover, if $k=t_{0}$, then $p=0$; if $k=t_{0}-2$, then $p>0$.

Case $1\left(t \in\left[2, t_{0}-1\right]\right)$. Since

$$
\begin{gathered}
f^{\prime}(t)>0 \text { for } t \in\left[2, t_{0}-1\right], \\
W(S(n, t)) \leq f(t) \leq f\left(t_{0}-1\right) .
\end{gathered}
$$

Moreover, since $W\left(S\left(n, t_{0}\right)\right)=\left(2 n^{2}-5 n+3\right)-(n-2) t_{0}-$ $\left(2 p k+p+t_{0} k^{2}\right)$ and $f\left(t_{0}-1\right)=\left(2 n^{2}-5 n+3\right)-(n-2) t_{0}+$ $(n-2)-\left(n-t_{0}\right)^{2} /\left(t_{0}-1\right)$, we have

$$
\begin{aligned}
\left(t_{0}-1\right) & \left(W\left(S\left(n, t_{0}\right)\right)-f\left(t_{0}-1\right)\right) \\
= & \left(n-t_{0}\right)^{2}-\left(2 p k+p+t_{0} k^{2}\right)\left(t_{0}-1\right) \\
& -(n-2)\left(t_{0}-1\right) \\
= & \left(1+t_{0} k+p\right)^{2}-\left(2 p k+p+t_{0} k^{2}\right)\left(t_{0}-1\right) \\
& -\left(t_{0}+t_{0} k+p-1\right)\left(t_{0}-1\right) \\
= & p^{2}+\left(4+2 k-2 t_{0}\right) p \\
& +\left(t_{0} k^{2}+3 t_{0} k+2 t_{0}-(k+1) t_{0}^{2}\right) \\
\geq & t_{0} k^{2}+3 t_{0} k+2 t_{0}-(k+1) t_{0}^{2} \\
= & t_{0}(k+1)\left(k+2-t_{0}\right) \geq 0 .
\end{aligned}
$$

Thus, $W\left(S\left(n, t_{0}\right)\right) \geq f\left(t_{0}-1\right)$, and together with (11), we have $W\left(S\left(n, t_{0}\right)\right) \geq W(S(n, t))$ for any $t \in\left[2, t_{0}-1\right]$. Observe from the above equation that $W\left(S\left(n, t_{0}\right)\right)=W(S(n, t))$ for some $t \in\left[2, t_{0}-1\right]$ if and only if $k=t_{0}-2, p=0$. But, this contradicts the fact that if $k=t_{0}-2$, then $p=0$. It follows that $W\left(S\left(n, t_{0}\right)\right)>W(S(n, t))$ for any $t \in\left[2, t_{0}-1\right]$.

Case $2\left(t \in\left[t_{0}+1, n-1\right)\right)$. Since $f^{\prime}(t)<0$ for $t \in\left[t_{0}+1, n\right)$, $W(S(n, t)) \leq f(t) \leq f\left(t_{0}+1\right)$. Since $f\left(t_{0}+1\right)=\left(2 n^{2}-5 n+\right.$ $3)-(n-2) t_{0}-(n-2)-\left(n-t_{0}-2\right)^{2} /\left(t_{0}+1\right)$, we have

$$
\begin{aligned}
\left(t_{0}+1\right)\left[W\left(S\left(n, t_{0}\right)\right)-f\left(t_{0}+1\right)\right] \\
=\left(n-t_{0}-2\right)^{2}+(n-2)\left(t_{0}+1\right) \\
\quad-\left(2 p k+p+t_{0} k^{2}\right)\left(t_{0}+1\right) \\
=\left(t_{0} k+p-1\right)^{2}+\left(t_{0}+t_{0} k+p-1\right)\left(t_{0}+1\right) \\
\quad-\left(2 p k+p+t_{0} k^{2}\right)\left(t_{0}+1\right) \\
=p^{2}-(2+2 k) p+t_{0}(k+1)\left(t_{0}-k\right) \\
=(p-k-1)^{2}+(k+1)\left(t_{0}^{2}-t_{0} k-k-1\right) .
\end{aligned}
$$

Recall that $t_{0}-2 \leq k \leq t_{0}$ and $0 \leq p \leq t_{0}-1$. Since

$$
t_{0}^{2}-t_{0} k-k-1= \begin{cases}t_{0}+1, & \text { if } k=t_{0}-2, \\ 0, & \text { if } k=t_{0}-1, \\ -t_{0}-1, & \text { if } k=t_{0},\end{cases}
$$

and the fact that if $k=t_{0}$, then $p=0$, we have

$$
\begin{aligned}
\left(t_{0}+1\right) & {\left[W\left(S\left(n, t_{0}\right)\right)-f\left(t_{0}+1\right)\right] } \\
& =(p-k-1)^{2}+(k+1)\left(t_{0}^{2}-t_{0} k-k-1\right) \geq 0,
\end{aligned}
$$

with equality only if $k=t_{0}$. It follows that $W(S(n, t)) \leq f(t) \leq$ $f\left(t_{0}+1\right) \leq W\left(S\left(n, t_{0}\right)\right)$, with $W(S(n, t))=W\left(S\left(n, t_{0}\right)\right)$ if and only if $t=t_{0}+1$ and $n=1+t_{0}+t_{0}^{2}$. The proof is completed.

\section{Acknowledgments}

The research was supported by KPCME (no. 210243), NSFC (no. 11161046), and the Tianyuan Special Fund of NSFC (no. 11126113).

\section{References}

[1] A. A. Dobrynin, R. Entringer, and I. Gutman, "Wiener index of trees: theory and applications," Acta Applicandae Mathematicae, vol. 66, no. 3, pp. 211-249, 2001.

[2] M. Aouchiche and P. Hansen, "Automated results and conjectures on average distance in graphs," in Graph Theory in Paris, Trends in Mathematics, pp. 21-36, Birkhäuser, Basel, Switzerland, 2007.

[3] F. Buckley and F. Harary, Distance in Graphs, Addison-Wesley, Redwood City, Calif, USA, 1990.

[4] A. Chen and F. Zhang, "Wiener index and perfect matchings in random phenylene chains," MATCH, vol. 61, no. 3, pp. 623-630, 2009.

[5] D. Li, B. Wu, X. Yang, and X. An, "Nordhaus-Gaddum-type theorem for Wiener index of graphs when decomposing into three parts," Discrete Applied Mathematics, vol. 159, no. 15, pp. 1594-1600, 2011.

[6] S. G. Wagner, "A class of trees and its Wiener index," Acta Applicandae Mathematicae, vol. 92, pp. 15-20, 2006.

[7] S. Wagner, "On the Wiener index of random trees," Discrete Mathematics, vol. 312, no. 9, pp. 1502-1511, 2012.

[8] B. Wu, "Wiener index of line graphs," MATCH, vol. 64, no. 3, pp. 699-706, 2010.

[9] S. Yamaguchi, "A note on Wiener index," MATCH, vol. 60, no. 2, pp. 645-648, 2008.

[10] Z. You and B. Liu, "Note on the minimal Wiener index of connected graphs with $n$ vertices and radius $r$," MATCH, vol. 66, no. 1, pp. 343-344, 2011.

[11] L. Zhang and B. Wu, "The Nordhaus-Goddum-type inequalities for some chemical indices," MATCH, vol. 54, no. 1, pp. 189-194, 2005.

[12] X.-D. Zhang, Q.-Y. Xiang, L.-Q. Xu, and R.-Y. Pan, “The Wiener index of trees with given degree sequences," MATCH, vol. 60, no. 2, pp. 623-644, 2008. 
[13] J. Plesník, "On the sum of all distances in a graph or digraph," Journal of Graph Theory, vol. 8, no. 1, pp. 1-21, 1984.

[14] E. DeLaViña and B. Waller, "Spanning trees with many leaves and average distance," Electronic Journal of Combinatorics, vol. 15, no. 1, article R33, p. 16, 2008.

[15] P. Erdős, M. Sakes, and V. T. Sós, "Maximum indueced trees in graphs," Journal of Combinatorial Theory, Series B, vol. 41, no. 1, pp. 61-79, 1986. 


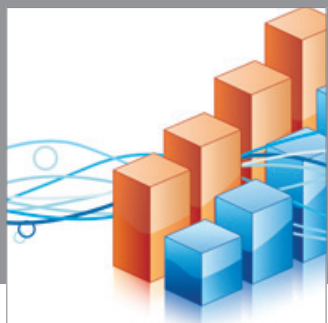

Advances in

Operations Research

mansans

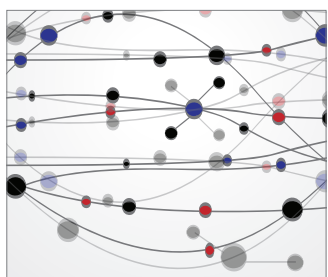

The Scientific World Journal
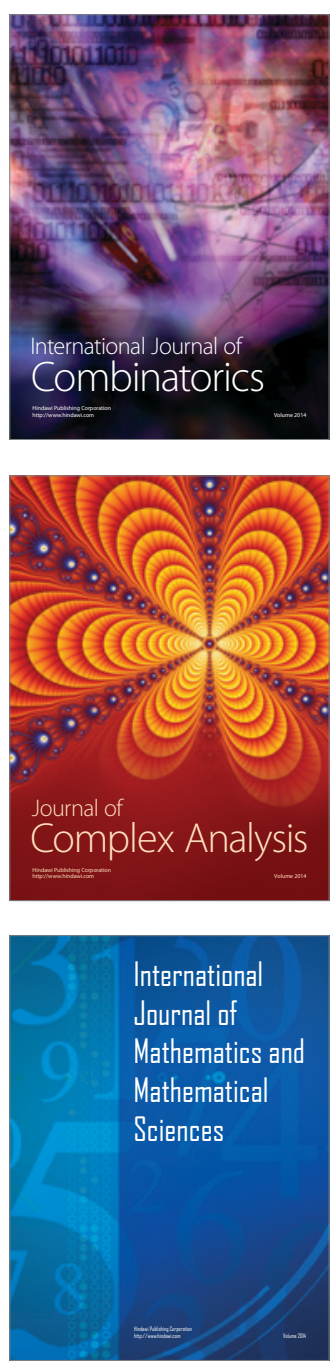
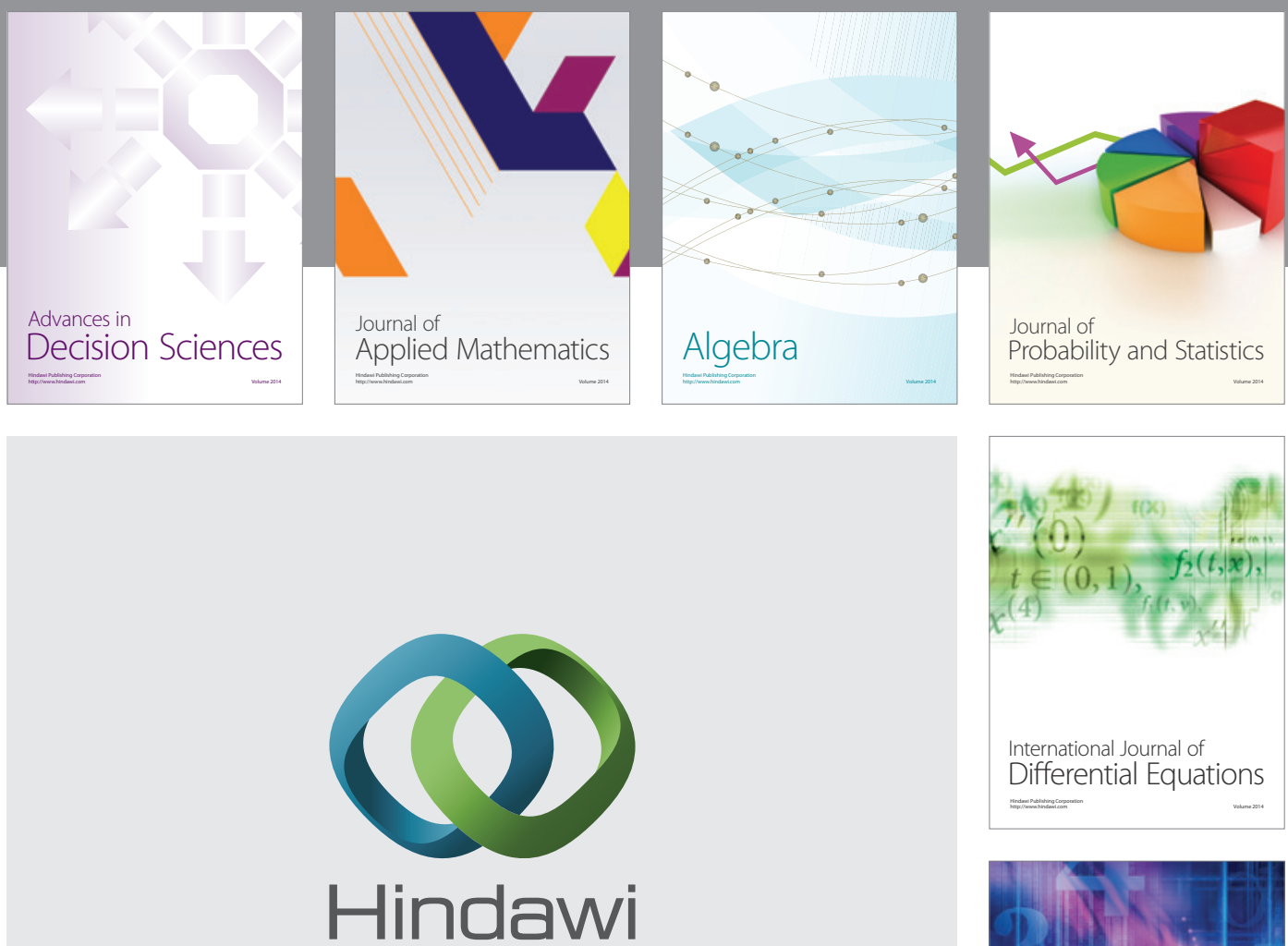

Submit your manuscripts at http://www.hindawi.com
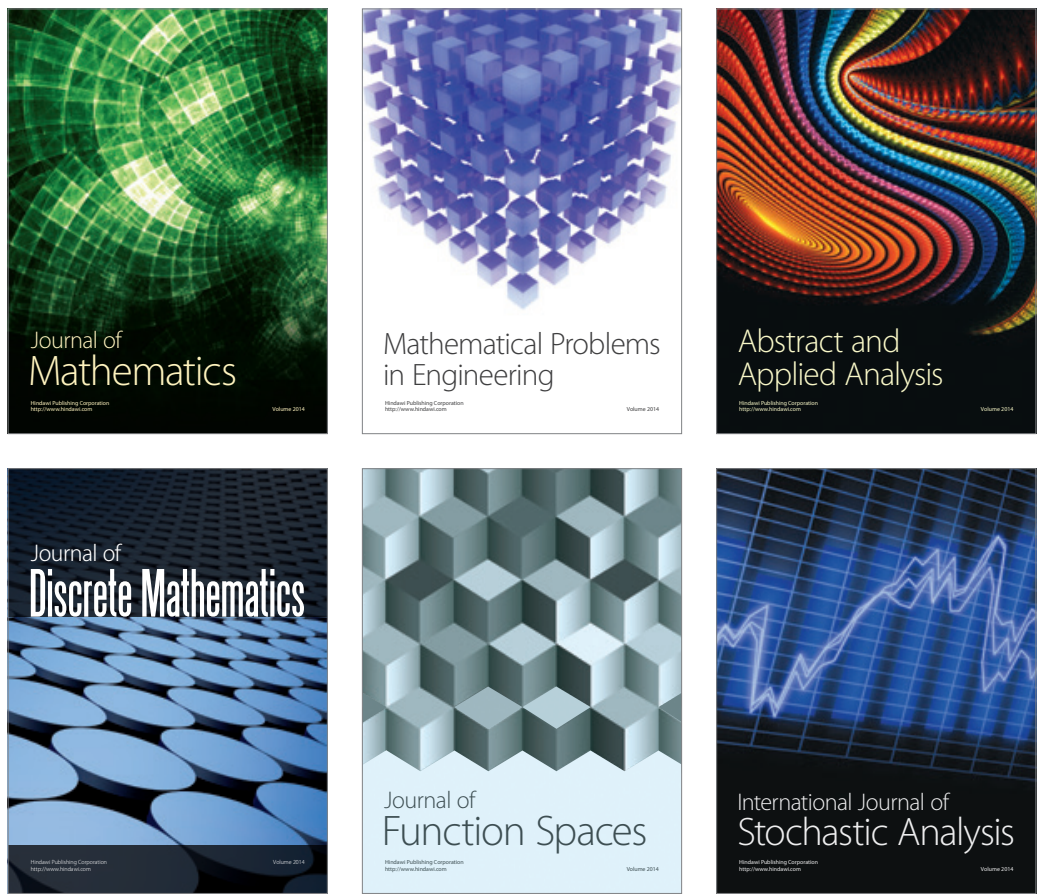

Journal of

Function Spaces

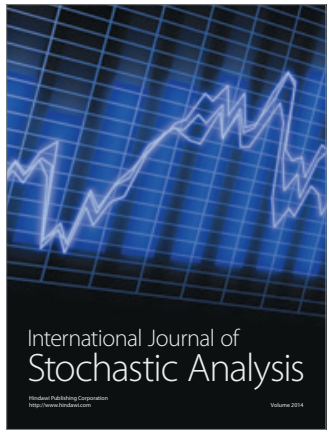

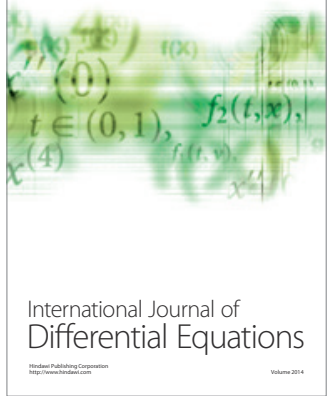
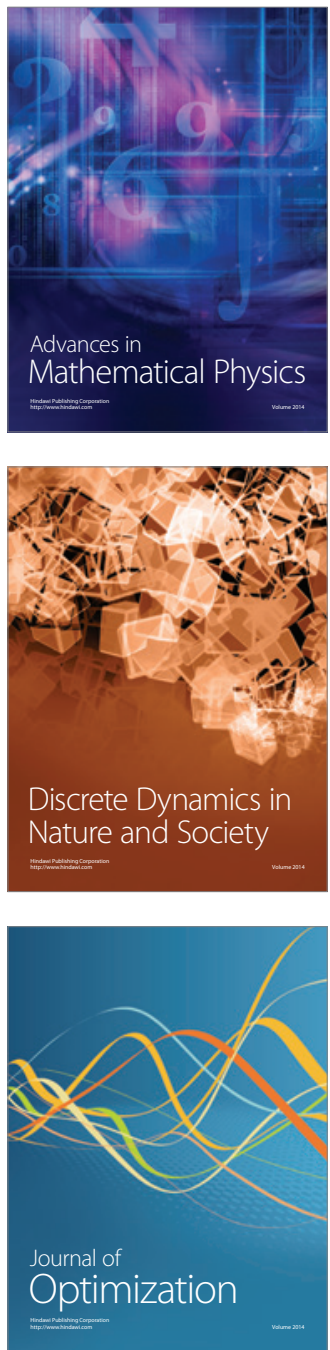